\author{
Submitted: \\ 03.10 .2020 \\ Accepted: \\ 09.11.2020 \\ Published: \\ 08.03.2021

\section{Dorsal scapular nerve entrapment neuropathy managed by ultrasound-guided hydrodissection - a case report} \\ Gaurav Kant Sharma1, Rajesh Botchu² \\ ${ }^{1}$ Department of Radiology, MGM Medical College and Hospital, Jaipur, India \\ ${ }^{2}$ Department of Musculoskeletal Radiology, Royal Orthopaedic Hospital, Birmingham, UK \\ Correspondence: Dr Rajesh Botchu, Department of Musculoskeletal Radiology, The Royal \\ Orthopaedic Hospital, Bristol Road South, Northfield, Birmingham, UK; tel.: 00441216854000, \\ e-mail:drbrajesh@yahoo.com \\ DOI: $10.15557 / \mathrm{JoU} .2021 .0012$

\section{Keywords \\ dorsal scapular nerve, \\ entrapment, hydrodissection}

\begin{abstract}
There are myriad causes for upper thoracic and interscapular pain which include pathologies involving the thoracic spine, costovertebral joints, facet joints and muscles (rhomboids and levator scapulae). Dorsal scapular nerve entrapment and the resulting neuropathy is a rare case of upper thoracic and medial scapular pain. The symptoms of dorsal scapular nerve entrapment neuropathy can be similar to other pathologies mentioned above. We report a rare case of dorsal scapular nerve entrapment managed successfully by ultrasound-guided hydrodissection, and describe the technique for the same. Awareness of this rare entity is
\end{abstract} \\ advised while evaluating upper thoracic and interscapular pain.
}

\section{Introduction}

There are a myriad of causes for upper thoracic and medial scapular pain. Dorsal scapular nerve entrapment neuropathy (DSNEN) is a rare cause of this symptom ${ }^{(1,2)}$. We report a case of dorsal scapular nerve entrapment neuropathy that was managed successfully with ultrasound-guided hydrodissection. Based on our knowledge, we believe this to be the first such case reported in the literature.

\section{Case report}

A 40-year-old male presented with 3 years' history of unilateral localised pain with paraesthesia along the medial border of the left scapula. He was a painter and used to play overhead sports (badminton). His past medical history was unremarkable, and there was no history of trauma or surgery at this site. On clinical examination, there was no winging of the scapula. MRI (magnetic resonance imaging) of the thoracic spine and chest was unremarkable. The patient subsequently had a high-resolution ultrasound scan on Siemens Acuson NX3 Elite Ultrasound Machine using 5-16 linear probe, which demonstrated significant thickening of the dorsal scapular nerve at the level of the belly of the rhomboid major muscle, with narrowing and flattening distally (Fig. 1). On that basis, the diagnosis of DSNEN at the level of the rhomboid major muscle belly was made. In view of the chronicity of the symptoms and failure of physiotherapy, which had been performed for 2 months, hydrodissection of the dorsal scapular nerve was considered at the level of entrapment.

\section{Technique of perineural hydrodissection}

Siemens Acuson NX3 Elite Ultrasound Machine with 6-15 MHz linear transducer is placed perpendicular to the superomedial border of the scapula. The dorsal scapular artery is identified deep to the rhomboid muscles, parallel and along the medial border of the scapula using power Doppler or colour Doppler. The dorsal scapular artery lies between the serratus posterior and the rhomboid major and minor muscles. The dorsal scapular nerve is identified as a small oval hypoechoic structure just medial to the dorsal scapular artery terminating at the T7 spinous process level. Once the nerve was identified and the site of entrapment was visualised, the needle trajectory used was planned medially to laterally in the plane along the long axis of the probe and using a $22 \mathrm{G}$ spinal needle hydrodissection with a total of $12 \mathrm{ml}$ of injectate $(4 \mathrm{ml}$ of $1 \%$ lignocaine $+7 \mathrm{ml}$ of $5 \%$ dextrose and $1 \mathrm{ml}$ of injection triamcinolone (40 mg) (Fig. 2). 


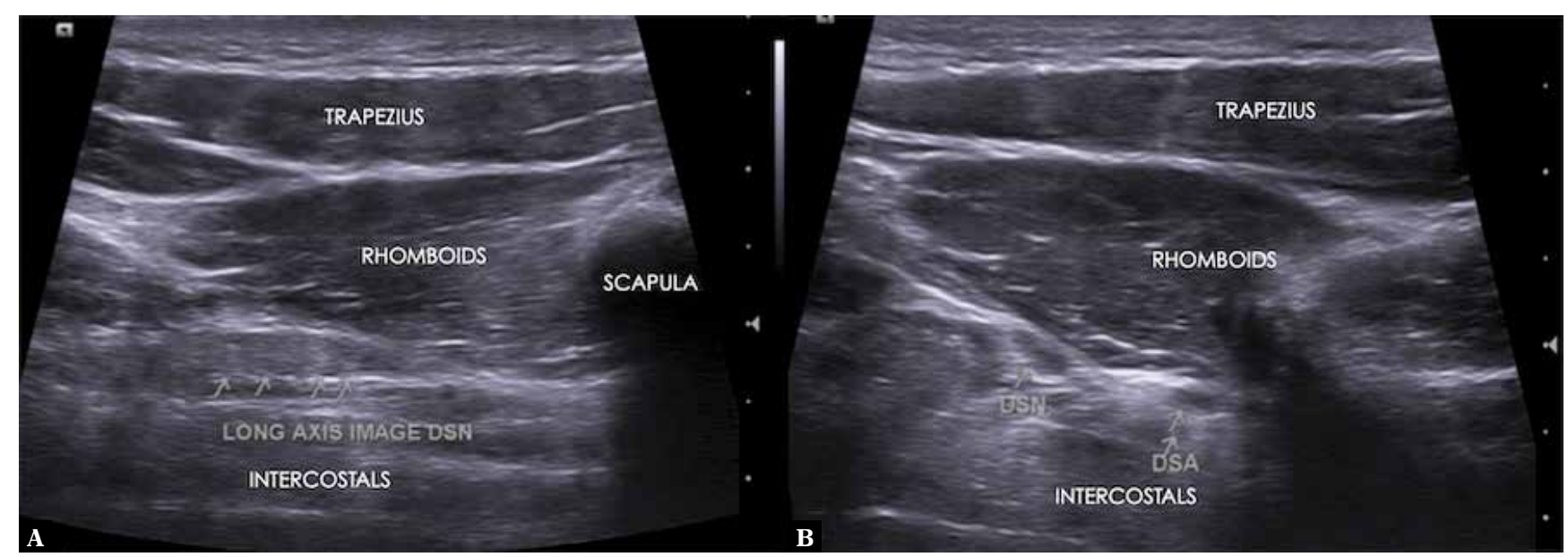

Fig. 1. Longitudinal (A) and axial (B) ultrasound image showing the dorsal scapular nerve (DSN), dorsal scapular artery (DSA), and adjacent muscles

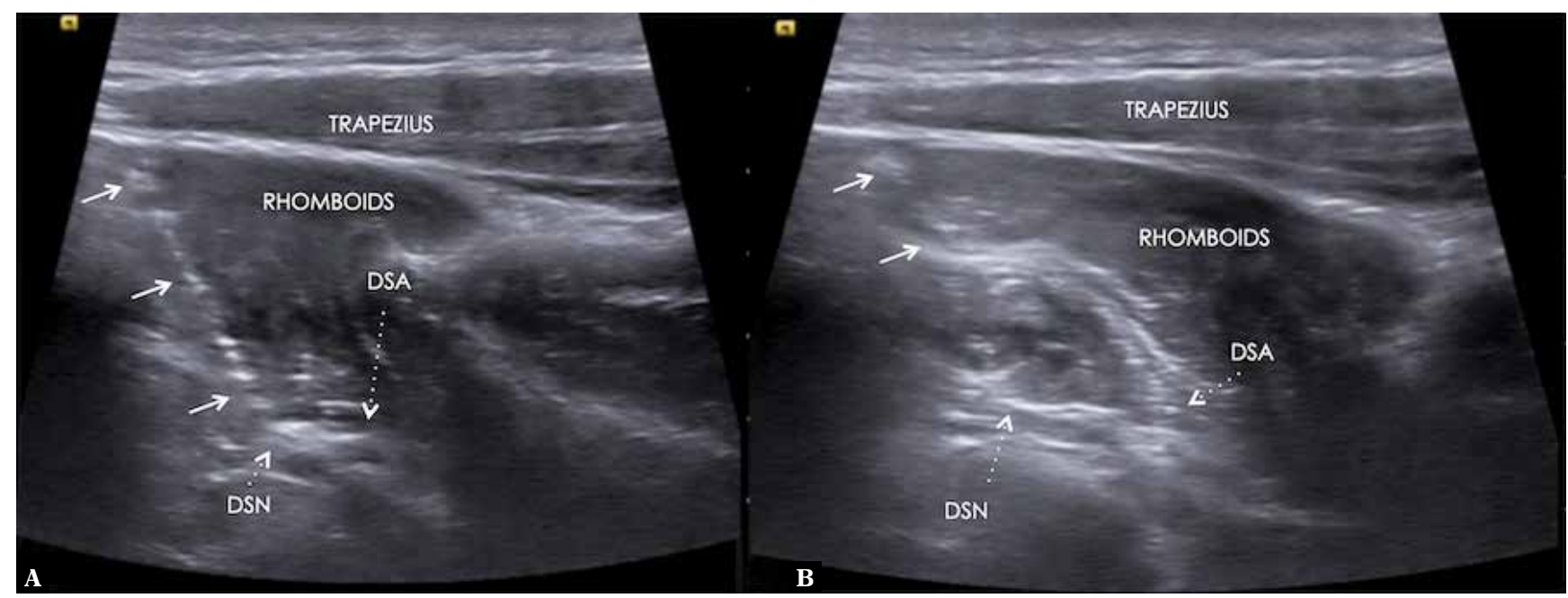

Fig. 2. Longitudinal ultrasound image showing the needle (arrow) with tip (A) adjacent to DSN and injectate adjacent to the DSN (B)

Perineural hydrodissection was done at the site of entrapment, where the change in nerve calibre was seen and circumferential 360-degree dissection of the dorsal scapular nerve was achieved. The pre-procedural VAS (visual analogue pain score) score was 6 . Immediately after the procedure, the patient's VAS score was 0/1. At 4 weeks' follow-up, the VAS score was 2, and using same injection volume and injectate, perineural hydrodissection was repeated. At 4 weeks' follow-up after the second injection, the patient reported complete resolution of symptoms and remained pain-free at 16 weeks' follow- up after the second injection.

\section{Discussion}

DSN arises from C5 (the superior branch of the brachial plexus) and C4 (in some cases). The nerve pierces the scalene medius at three-fourths before coursing posteroinferiorly between the trapezius and levator scapula, and subsequently between the rhomboids and serratus anterior along the medial border of the scapula (Fig. 3). It supplies the middle scalene levator scapula and rhomboid (major and minor). The most common site of entrapment is in the scalene medius ${ }^{(1)}$. The abnormalities can be seen in overhead players (athletes and volleyball players) and workers (painters, electricians). DSN injuries can also result as sequelae including anterior dislocation of the shoulder and can be iatrogenic. DSN injury or neuropathy may result in scapular winging(1).

The symptoms of DSNEN include pain and dysaesthesia with or without atrophy along the medial border of the scapula and the upper thoracic region. Associated winging of the scapula can be present in $17 \%$ of cases $^{(1,2)}$. The diagnosis is often made by exclusion. One should consider the diagnosis if imaging of the spine and chest are unremarkable or if the pain is exclusively along the medial border of the scapula.

DSN neuropathies are common in females (4:1 female to male ratio), with a peak incidence in the $3^{\text {rd }}$ and $4^{\text {th }}$ decades of life. These are unilateral in the majority of patients $^{(1)}$. 


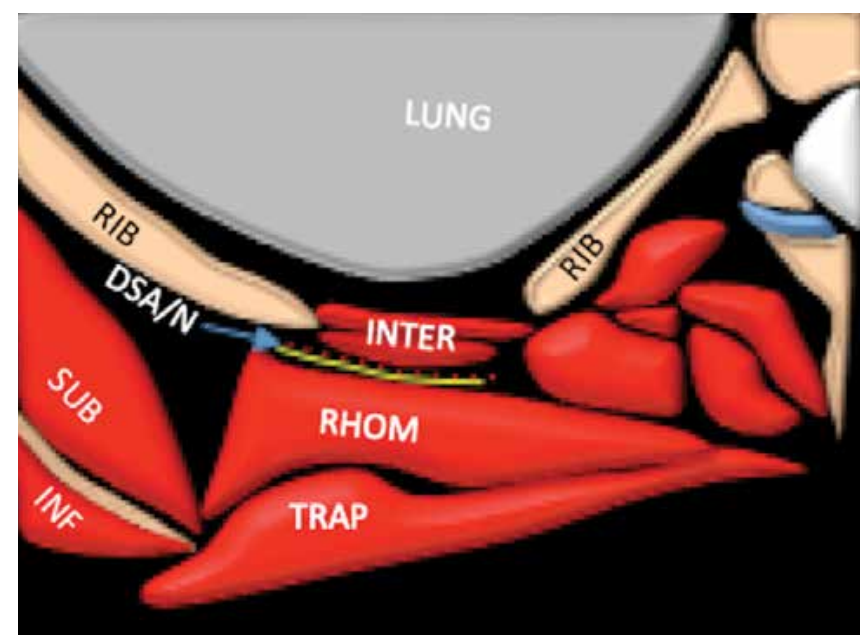

Fig. 3. Schematic anatomy of the posterior chest wall showing the DSN, DSA, rhomboids (RHOM), intercostal muscles (INTER), subscapularis (SUB), infraspinatus (INF)

High-resolution ultrasound is able to demonstrate the course of the nerve, evaluating for thickening, oedema, and an increased signal within the nerve on Doppler ${ }^{(3)}$. These can be analysed by static and dynamic examination. It can also demonstrate the site of entrapment or compression of the nerve. Proximal to the site of the entrapment, the nerve is thickened, with thinning or flattening distally. This can be absent in some patients with chronic neuropathy. In such cases, the phenomenon of 'double crush' (compression/ entrapment at more than one site) should be excluded ${ }^{(4)}$.

Ultrasound-guided hydrodissection of the peripheral nerve is an effective and safe technique. During entrapment, there is change in the morphology of the nerve resulting in alteration/interruption of the flow of axoplasm with some eventual fibrosis resulting in increased calibre of the nerve proximal to the site of nerve entrapment and flattening at the site of entrapment.

\section{References}

1. Muir B: Dorsal scapular nerve neuropathy: a narrative review of the literature. J Can Chiropr Assoc 2017; 61: 128-144.

2. Sultan HE, Younis El-Tantawi GA: Role of dorsal scapular nerve entrapment in unilateral interscapular pain. Arch Phys Med Rehabil 2013; 94: 1118-1125.
Hydrodissection of a nerve helps to improve its kinematic properties and relieve the entrapment ${ }^{(4)}$.

This is now considered one of the mainstays of treatment of compressive/entrapment neuropathy. Evan et al. showed a significant improvement in the gliding of the median nerve after hydrodissection with $5 \mathrm{ml}$ of saline in cadavers. Wu et al. noticed a significant improvement with the same in a randomised controlled trial. Hydrodissection can be performed with normal saline, local anaesthetic, 5\% dextrose with or without steroids ${ }^{(3,4)}$.

In our case, an injectate of $1 \%$ lidocaine, $5 \%$ dextrose and triamcinolone was used for hydrodissection, with complete resolution of symptoms at 4 months' follow-up.

\section{Conclusion}

Dorsal scapular nerve entrapment neuropathy is a rare case of upper thoracic and medial scapular pain that can be managed successfully with ultrasound-guided hydrodissection, as demonstrated in this particular case.

\section{Declaration}

Informed consent obtained from patient.

\section{Conflict of interest}

Authors do not report any financial or personal connections with other persons or organizations, which might negatively affect the contents of this publication and/or claim authorship rights to this publication.

3. Chang KV, Wu WT, Özçakar L: Ultrasound imaging and guidance in peripheral nerve entrapment: hydrodissection highlighted. Pain Manag 2020; 10: 97-106.

4. Cass SP: Ultrasound-guided nerve hydrodissection: what is it? A review of the literature. Curr Sports Med Rep 2016; 15: 20-22. 